\title{
One Type of Optimal Portfolio Selection in Birandom Environments
}

\author{
Limei Yan \\ Mathematics Department, Dezhou University \\ Shandong 253023, China \\ E-mail: yanlimei9898@163.com
}

\begin{abstract}
In order to solve the portfolio problem when security returns are birandom variables, firstly we propose a new definition of risk, then one type of portfolio selection based on expected value and risk is provided according to birandom theory. Furthermore, A hybrid intelligent algorithm by integrating birandom simulation and genetic algorithm is designed. Finally, one numerical experiment is provided to illustrate the effectiveness of the hybrid intelligent algorithm.
\end{abstract}

Keywords: Birandom variable, Portfolio selection, Expected value operator, Confidence curve, Hybrid intelligent algorithm

\section{Introduction}

The theory of portfolio selection was initially provided by Markowitz $(1952$, p.77) and has been greatly developed since then. It is concerned with selecting a combination of securities among portfolios containing large number of securities to reach the goal of obtaining satisfactory investment return. In his path-break work, Markowitz proposed a principle that when making investment decision, an investor should always strike a balance between maximizing the return and minimizing the risk, i.e., the investor maximize return for a given level of risk, or one should minimize risk for a predetermined return level. More importantly, Morkowitz initially quantified investment return as the expected value of returns of securities, and risk as variance from the expected value. After Maokowitz's work, scholars have been showing great enthusiasm in portfolio management, trying different mathematical approaches to develop the theory of portfolio selection. Traditionally, returns of individual securities were assumed to be stochastic variables, and many researchers were focused on extending Markowitz's mean-variance models and on developing new mathematical approaches to solve the problems of computation. Peng (2007,p.433) proposed concept of birandom variable and the framework of birandom programming. However, investors may come across birandom returns in portfolio selection situations. For example, security returns are usually regarded to be normally distributed random variables, but the expected value may be still random variable, thus investors have to face random returns with random parameters, to deal with this type of uncertainty, we propose the security returns could be regarded as birandom variables. As a general mathematical description for this kind of stochastic phenomenon with incomplete statistical information, birandom variable is defined as a mapping with some kind of measurability from a probability space to a collection of random variables.

In general, there are three types of risk definitions in portfolio selection problems. Variance is the earliest and most commonly accepted definition of risk for portfolio selection initially proposed by Markowitz (1952, p.77). A variety of extensions to Markowitz's mean-variance models has been proposed. Semivariance is the second type of risk definitions, and was also proposed by Markowitz (1959). Semivariance is an improvement of variance because semivariance only measures portfolio return below the expected value. Many models have been built to minimize semivariance in different cases. The third popular definition of risk is a probability of a bad outcome initially by Roy (1952, p.431). Much research has been undertaken to find ways of minimizing the probability of the bad outcome. Recently, Huang (2007, p.5404) proposed another new definition of risk for portfolio selection in fuzzy and random fuzzy environments. The detailed exposition on the definition of risk had been recorded in the literature, the interested readers may consult it. We can regard it as the fourth type of risk. Her work has enriched the risk theory for portfolio selection. We try to do something for portfolio selection in birandom environments, and give a new risk definition and a model for portfolio selection according to the proposed risk.

The rest of this paper is arranged as follows. After reviewing some necessary knowledge about birandom variable in section 2, in section 3, one type of risk for portfolio selection model under birandom environment is proposed. In section 4, we give a model for portfolio selection from the point of the new definition of risk. To provide a general method for solving the new models, in section 5, a hybrid intelligent algorithm integrating genetic algorithm and birandom simulation is designed. To better illustrate the modeling idea and demonstrate the effectiveness of the proposed algorithm, one numerical example is provided in section 6 . 


\section{Preliminaries}

Birandom variable theory was introduced by Peng (2007, p.433). To better understand the proposed model for portfolio selection, let us briefly review some necessary knowledge about birandom variable.

Definition 1 A birandom variable $\xi$ is a mapping from a probability space $(\Omega, A, \operatorname{Pr}$ ) to a collection of random variables such that for any Borel subset $B$ of the real line $R$, the induced function $\operatorname{Pr}\{\xi(\omega) \in B\}$ is a measurable function with respect to $\omega$.

Example 1 Let $\Omega=\left\{\omega_{1}, \omega_{2}\right\}$, and $\operatorname{Pr}\left\{\omega_{1}\right\}=\operatorname{Pr}\left\{\omega_{2}\right\}=1 / 2$. Assume that $\xi$ is a function on $(\Omega, \mathrm{A}, \operatorname{Pr})$ as follows.

$\xi(\omega)= \begin{cases}\xi_{1}, & \omega=\omega_{1} \\ \xi_{2}, & \omega=\omega_{2}\end{cases}$

where $\xi_{1}$ is a uniformly distributed random variable on $[0,1]$ and $\xi_{2}$ is a normally distributed random variable with mean 0 and variance 1 , i.e., $\xi_{1} \sim U[0,1]$ and $\xi_{2} \sim N(0,1)$. Then $\xi$ is a birandom variable according to the definition.

The following are the definitions of the expected value operator and variance of birandom variance and the primitive chance of birandom event.

Definition 2 (Peng (2007, p.4330)). Let $\xi$ be a birandom variable defined on the probability space $(\Omega, A, \operatorname{Pr})$. Then the expected value of birandom variable $\xi$ is defined as

$$
E[\xi]=\int_{0}^{\infty} \operatorname{Pr}\{\omega \in \Omega \mid E[\xi(\omega)] \geq t\} \mathrm{dt}-\int_{-\infty}^{0} \operatorname{Pr}\{\omega \in \Omega \mid E[\xi(\omega)] \leq t\} \mathrm{dt}
$$

provided that at least one of the above two integrals is finite.

Definition 3 (Peng (2007, p.4330)). Let $\xi=\left(\xi_{1}, \xi_{2}, \cdots, \xi_{n}\right)$ be a birandom vector on $(\Omega, \mathrm{A}, \operatorname{Pr})$, and $f: R^{n} \rightarrow R^{m}$ be a vector-valued Borel measurable function. Then the primitive chance of birandom event characterized by $f(\xi) \leq 0$ is a function from $(0,1]$ to $[0,1]$, defined as

$\operatorname{Ch}\{f(\xi) \leq 0\}(\alpha)=\sup \{\beta \mid \operatorname{Pr}\{\omega \in \Omega \mid \operatorname{Pr}\{f(\xi(\omega)) \leq 0\} \geq \beta\} \geq \alpha\}$.

Theorem 1 (Peng (2007, p.4330)). Let $\xi=\left(\xi_{1}, \xi_{2}, \cdots, \xi_{n}\right)$ be a birandom vector on $(\Omega, \mathrm{A}, \operatorname{Pr})$, and $f: R^{n} \rightarrow R^{m}$ be a Boral measurable function. Then the chance

$\operatorname{Ch}\{f(\xi) \leq 0\}(\alpha)$

is a decreasing and left-continuous function of $\alpha$.

Theorem 2 (Peng (2007, p.4330)). Let $\xi$ be a birandom variable and $\alpha$ a given number in $(0,1]$, then the chance distribution

$\mathrm{Ch}\{\xi \geq x\}(\alpha)$ is a decreasing and left-continuous function of $x$.

\section{New definition of risk}

In reality, some investors are only sensitive to one preset bad case. They regard as safe those securities whose chance of this bad case occurring is lower than the investors' tolerance level. Other investors consider all the possible unfavorable cases, and only those securities whose chance of every unfavorable case occurring is lower than the investors' tolerance level are regarded as safe. We will define the risk from this perspective.

Definition 4 Let $\xi$ be a birandom variable on the probability space $(\Omega, \mathrm{A}, \operatorname{Pr})$, and $\delta$ the preset confidence level and $b$ the target return. Then the curve $f(\delta, r)=\operatorname{Ch}\{b-\xi \geq r\}(\delta), \forall r \in R$ is called the risk curve of an 
investment in the portfolio, and $r$ the loss severity indicator.

The greater the indicator $r$, the more severe the loss $b-\xi$. The risk curve $f(\delta, r)$ gives the chance of the occurrence of all events when the birandom return $\xi$ is $r$ less than the target return $b$.

From theorem 1 and theorem 2 we can derive that the risk curve $f(\delta, r)$ is a decreasing function with respect to $\delta$ and $r$, that is, the greater the $\delta$, the smaller the $f(\delta, r)$ value, the greater the $r$, the smaller the $f(\delta, r)$.

To determine whether a portfolio selection is risky, an investor must first decide what is his or her maximum tolerance level of each bad event occurring. Usually, the worse the event, the lower the tolerance level. Then for every loss severity indicator $r_{0} \in R$, the investor always can give a confidence level corresponding $\alpha\left(r_{0}\right)$, thus the confidence level $\alpha(r)$ is a function of the loss severity indicator $r$, the function $\alpha(r)$ is called the confidence curve.

Let $\xi$ be a birandom return of a portfolio $A$, and $\alpha(r)$ the confidence curve. We can say that $A$ is safe if $f(\delta, r)=\operatorname{Ch}\{b-\xi \geq r\}(\delta) \leq \alpha(\mathrm{r})$ for every $r \in R$, where $b$ is the target return and $\delta$ the preset credibility level. The number $r$ denotes all possible loss severity indicator. If the investor is only concern with one special loss severity indicator $r_{0}$, then the risk becomes the chance $\mathrm{Ch}\left\{b-\xi \geq r_{0}\right\}(\delta)$, Which is exactly the ordinary chance measure of birandom variable.

\section{Birandom portfolio selection}

Let us select a portfolio according to the definition of risk in the preceding content. Let $x_{i}$ denotes the investment proportions in security $i, \xi_{i}$ the birandom return for the $i$ th security, $i=1,2, \cdots, n$, respectively. Let $r$ denote the loss severity indicator, and $\alpha(r)$ the confidence curve preset by the investor. To obtain the maximum investment return and avoid risk, the investor should select an optimal combination of securities from the portfolio safe point. We use the expected value of the securities to express the investment return. Thus we should set a goal of maximizing the expected return of a portfolio, and require that the risk curve $f(\delta, r)$ is not larger than the confidence curve $\alpha(r)$. Let $b$ be the target return and $\delta$ the preset credibility level. Then the model is formulated as follows:

$$
\begin{aligned}
& \max _{x} E\left[\sum_{i=1}^{n} x_{i} \xi_{i}\right] \\
& \text { subject to } \\
& \mathrm{Ch}\left\{b-\sum_{i=1}^{n} x_{i} \xi_{i} \geq r\right\}(\delta) \leq \alpha(r), \forall r \geq 0 \\
& \sum_{\mathrm{i}=1}^{n} x_{i}=1 \\
& x_{i} \geq 0, i=1,2, \cdots, n
\end{aligned}
$$

When the birandom returns degenerate to random, the chance constraint becomes

$\operatorname{Pr}\left\{b-\sum_{i=1}^{n} \xi_{i} x_{i} \geq r\right\} \leq \alpha(r), \forall r \geq 0$,

thus the model is the following

$$
\begin{aligned}
& \max _{x} E\left[\sum_{i=1}^{n} x_{i} \xi_{i}\right] \\
& \text { subject to } \\
& \quad \operatorname{Pr}\left\{b-\sum_{i=1}^{n} x_{i} \xi_{i} \geq r\right\} \leq \alpha(r), \forall r \geq 0 \\
& \quad \sum_{i=1}^{n} x_{i}=1 \\
& \quad x_{i} \geq 0, i=1,2, \cdots, n
\end{aligned}
$$

Furthermore, if the investor only concerns one preset loss severity level $r_{0}$, then the model (2) can be converted into the formulation: 
$\max _{x} E\left[\sum_{i=1}^{n} x_{i} \xi_{i}\right]$

subject to

$$
\begin{aligned}
& \operatorname{Pr}\left\{b-\sum_{i=1}^{n} x_{i} \xi_{i} \geq r_{0}\right\} \leq \alpha\left(r_{0}\right) \\
& \sum_{\mathrm{i}=1}^{n} x_{i}=1 \\
& x_{i} \geq 0, \quad i=1,2, \cdots, n
\end{aligned}
$$

\section{Hybrid intelligent algorithm}

Since the two-fold uncertainty of birandom variable, it is difficult to analytically solve the models (1), (2) and (3). To provide a general solution to the models, we design a hybrid intelligent algorithm integrating genetic algorithm (GA) and birandom simulation. Roughly speaking, in the proposed hybrid intelligent algorithm, the technique of birandom simulation is applied to compute the expected value and the chance measure, then birandom simulation and GA are integrated for solving the birandom models.

\subsection{Birandom simulation}

In this section, we first discuss the calculation of the expected value and the chance measure of birandom variables.

Let $\xi_{i}$ be birandom variables and $x_{i}$ decision variables, $i=1,2, \cdots, n$, respectively. Write $f(x, \xi)=\sum_{i=1}^{n} x_{i} \xi_{i}$, where $x=\left(x_{1}, x_{2}, \cdots, x_{n}\right), \xi=\left(\xi_{1}, \xi_{2}, \cdots, \xi_{n}\right)$. Let $b$ be the target return and $\delta$ the preset credibility level. The number $r$ denotes all possible loss severity indicator. In order to solve the proposed models, we must handle the following two types of uncertain function.

$U_{1}: x \rightarrow E\{f(x, \xi)\}$

$U_{2}: x \rightarrow \operatorname{Ch}\{b-f(x, \xi) \geq r\}(\delta) \geq \alpha(r), \forall r \geq 0$.

$U_{1}(x)$ may be estimated by the following procedure.

Algorithm 1 (birandom simulation for $U_{1}(x)$ )

Step 1. Set $E[f(x, \xi)]=0$.

Step 2. Generate $\omega$ from $\Omega$ according to the probability measure Pr.

Step 3. $E[f(x, \xi)] \leftarrow E[f(x, \xi)]+E[f(x, \xi(\omega))]$ may be calculated by stochastic simulation.

Step 4. Repeat the second to the third steps $N$ times.

Step 5. $E[f(x, \xi)] \leftarrow E[f(x, \xi)] / N$.

Algorithm 2 (birandom simulation for $U_{2}(x)$ )

Step 1. Set $l=1$.

Step 2. randomly generate a real number $r$ according to the confidence curve given by the investor.

Step3. Generate $\omega_{1}, \omega_{2}, \cdots, \omega_{N}$ from $\Omega$ according to the probability measure Pr.

Step 4. Compute the probability $\beta_{n}=\operatorname{Pr}\left\{b-f\left(x, \xi\left(\omega_{n}\right)\right) \geq r\right\}$ for $n=1,2, \cdots, N$, respectively, by stochastic simulation.

Step 5. Set $N^{\prime}$ as the integer part of $\delta N$.

Step 6. Return the $N^{\prime}$ th largest element $\bar{\beta}$ in $\left\{\beta_{1}, \beta_{2}, \cdots, \beta_{N}\right\}$.

Step 7. If $\bar{\beta}$ is no larger than $\alpha(r)$, then $l=l * 1$, else $l=l * 0$.

Step 8. repeat the second to the fifth steps for a given number times.

Step 9. If $l=1$, then return YES, else return NO.

Remark: here YES means that the investment proportion $x$ is feasible; NO means that $x$ is infeasible.

\subsection{Genetic algorithm}

Representation structure: A solution $x=\left(x_{1}, x_{2}, \cdots, x_{n}\right)$ is represented by the chromosome $V=\left(v_{1}, v_{2}, \cdots, v_{n}\right)$, where the genes $v_{1}, v_{2}, \cdots, v_{n}$ are randomly generated in the interval [0,1], and the relation between $x$ and $V$ are 
formulated as follows:

$x_{i}=v_{i} /\left(v_{1}+v_{2}+\cdots+v_{n}\right), i=1,2, \cdots, n$,

which ensures that

$x_{1}+x_{2}+\cdots+x_{n}=1, x_{i} \geq 0, i=1,2, \cdots, n$ always holds.

Initialization process: pop_size number of chromosomes are initialized randomly by generating points $\left(v_{1}, v_{2}, \cdots, v_{n}\right)$ from the hypercube $[0,1]^{n}$ pop-size times. Since the constraint required that $x=\left(x_{1}, x_{2}, \cdots, x_{n}\right)$ satisfy $x_{1}+x_{2}+\cdots+x_{n}=1$, based on the relation between $x$ and $V$, the feasibility of the randomly generated chromosomes is obvious.

Evaluation function: Evaluation function, denoted by $\operatorname{Eva}(V)$, is to assign a probability of reproduction to each chromosome $V$ so that its likelihood of being selected is proportional to its fitness relative to the other chromosomes in the population. That is, the chromosomes with higher fitness will have more chance to produce offspring by using roulette wheel selection. One well-known evaluation function is based on allocation of reproductive trial according to rank rather than actual objective values. We can rearrange the pop-size chromosomes according to their objective values to make better chromosome take smaller ordinal number. That is, after rearrange, among pop-size chromosomes $V_{1}, V_{2}, \cdots, V_{\text {pop_size }}, V_{1}$ is the best chromosome, and $V_{\text {pop_size }}$ the worst one, then a parameter $a \in(0,1)$ in the genetic system is given. We can define the rank-based evaluation function as follows:

$\operatorname{Eva}\left(V_{i}\right)=a(1-a)^{i}, i=1,2, \cdots$, pop_size.

Note that $i=1$ means the best individual, $i=p o p_{\_}$size the worst one.

Selection process: Firstly, calculate the cumulative probability $q_{i}$ for each chromosome $V_{i}$,

$q_{0}=0, q_{i}=\sum_{j=i}^{i} \operatorname{Eva}\left(V_{j}\right), i=1,2, \cdots$, pop_size

Secondly, generate a random number $r$ in $\left(0, q_{p o p_{-} \text {size }}\right]$, and select the chromosome $V_{i}$ if $r$ satisfies $q_{i-1}<r \leq q_{i}$.

Repeat the second and third steps pop_size times and obtain pop_size copies of chromosome.

Crossover operation: A parameter $p_{c}$ of a genetic system as the probability of crossover is defined first. The parents for crossover operation are selected by doing the following process repeatedly from $i=1$ to pop_size : Generating a random number $r$ from the interval [0,1], the chromosome $V_{i}$ is selected as a parent if $r<p_{c}$, the selected parents are denoted by $V_{1}^{\prime}, V_{2}^{\prime}, V_{3}^{\prime}, \cdots$ and divided into the pairs: $\left(V_{1}^{\prime}, V_{2}^{\prime}\right),\left(V_{3}^{\prime}, V_{4}^{\prime}\right),\left(V_{5}^{\prime}, V_{6}^{\prime}\right), \cdots$. The crossover operation on each pair is illustrated by $\left(V_{1}^{\prime}, V_{2}^{\prime}\right)$. At first, we generate a random number $c$ from the open interval $(0,1)$, then the operator on $V_{1}^{\prime}$ and $V_{2}^{\prime}$ will product two children $X$ and $Y$ as follows:

$X=c V_{1}^{\prime}+(1-c) V_{2}^{\prime}, \quad Y=(1-c) V_{1}^{\prime}+c V_{2}^{\prime}$.

If both children are feasible, then we replace the parents with them. If not, we keep the feasible one if it exists, and then redo the crossover operator by regenerating a random number $c$ until two feasible children are obtained or a given number of cycles is finished. In this case, we only replace the parents with the feasible children .

Mutation operation: A parameter $p_{m}$ of a genetic system as the probability of mutation is defined first. This probability gives us the expected number of $p_{m} \cdot p o p_{-}$size of chromosomes undergoing the mutation operations. We repeat the following steps from $i=1$ to $p o p_{-}$size: Generating a random number $r$ from the interval $[0,1]$, the chromosome $V_{i}$ is selected as a parent if $r<p_{m}$. For each selected parents $V_{i}$, we mutate it in the following way. Let $M$ be an appropriate large positive number. We choose a mutation direction $d$ in $R^{n}$ randomly. If $V+M \cdot d$ is not feasible, then we set $M$ as a random number between 0 and $M$ until it is feasible. If the above process cannot find a feasible solution in a predetermined number of iterations, then we set $M=0$. Anyway, we replace the parent $V_{i}$ with its feasible child $V+M \cdot d$.

The following is the hybrid intelligent algorithm integrating birandom simulation and genetic algorithm.

Algorithm 3 (hybrid intelligent algorithm)

Step 1 Initialize pop_size chromosomes.

Step 2 Calculate the objective values for all chromosomes by birandom simulation. 
Step 3 Given the rank order of the chromosomes according to the objective values, and the values of the rank-based evaluation function of the chromosomes.

Step 4 Compute the fitness of each chromosome according to the rank-based evaluation function.

Step 5 Select the chromosomes by spinning the roulette wheel.

Step 6 Update the chromosomes by crossover and mutation operations.

Step 7 Repeat the second step to the sixth step for a given number of cycles.

Step 8 Take the best chromosome as the solution of portfolio selection.

\section{Numerical example}

To illustrate the modeling idea and to test the effectiveness of the designed hybrid intelligent algorithm, let us consider one numerical example. The example is performed on a personal computer by using $\mathrm{C}++$ programming language. The parameters in the HIA are set as follows: the probability of crossover $p_{c}=0.3$, the probability of mutation $p_{m}=0.2$, the parameter $\alpha=0.05$ in the rank-based evaluation function.

Example 2 Assume that there are 5 securities, the returns of securities are all birandom variables.

$\max _{x} E\left[\sum_{i=1}^{5} x_{i} \xi_{i}\right]$

subject to

$$
\begin{aligned}
& \operatorname{Ch}\left\{b-\sum_{i=1}^{5} x_{i} \xi_{i} \geq r\right\}(\delta) \leq \alpha(r), r \geq 0 \\
& \sum_{\mathrm{i}=1}^{5} x_{i}=1 \\
& x_{i} \geq 0, i=1,2, \cdots, 5
\end{aligned}
$$

Where

$\xi_{1} \sim N\left(\mu_{1}, 1\right)$ with $\mu_{1} \sim U(0,1)$,

$\xi_{2} \sim N\left(\mu_{2}, 1\right)$ with $\mu_{2} \sim U(1,2)$,

$\xi_{3} \sim N\left(\mu_{3}, 1\right)$ with $\mu_{3} \sim U(2,3)$,

$\xi_{4} \sim N\left(\mu_{4}, 2\right)$ with $\mu_{4} \sim U(3,4)$,

$\xi_{5} \sim N\left(\mu_{5}, 1\right)$ with $\mu_{5} \sim U(4,5)$.

Suppose that the investor has given the confidence curve and other parameters :

$\alpha(r)=\frac{1}{(r+1.1)^{4}}, r \geq 0 . b=0.9, \delta=0.9$.

Here, $N\left(\mu, \sigma^{2}\right)$ represents the normally distributed random variable with mean $\mu$ and standard variance $\sigma$ and $U(a, b)$ denotes the uniform distribution on the interval $(a, b)$. A run of the hybrid intelligent algorithm with 3000 generations shows that among 5 securities, in order to gain maximum expected value of the total securities return the investor should assign his money according to the optimal solution:

$x_{1}{ }^{*}=0, x_{2}{ }^{*}=0.1325, x_{3}{ }^{*}=0.2236, x_{4}{ }^{*}=0.2782, x_{5}{ }^{*}=0.3657$.

\section{References}

A. Charnes \& W. W. Cooper. (1965). Chance-constrained programming. Management Science, 6, 73-79.

A. D. Roy. (1952). Safety first and the holding of assets. Econometrics, 20 ,431-449.

B. Liu. Uncertainty theory, 3rd edition. http://orsc.edu.cn/liu/ut.pdf.

H.Markowit z. (1952). Portfolio selection. Journal of Finance , 7, 77-91.

H.Markowitz. (1959). Portfolio selection: Efficient diversification of investment, Wiley, New York.

Jin peng. (2007). Birandom variables and birandom programming. Computer \& Industrial Engineering, 53, 433-453.

Xiaoxia Huang. (2006). Fuzzy chance-constrained portfolio selection. Applied mathematics and computation, 177, 500-507.

Xiaoxia Huang. (2007). A new perspective for optimal portfolio selection with random fuzzy returns. Information science ,177, 5404-5414. 